\title{
AN OPEN-CIRCUIT-VOLTAGE MODEL OF LITHIUM-ION BATTERIES FOR EFFECTIVE INCREMENTAL CAPACITY ANALYSIS
}

\author{
Caihao Weng* \\ Department of Naval Architecture \\ and Marine Engineering \\ University of Michigan \\ Ann Arbor, Michigan 48109 \\ Email: chsweng@umich.edu
}

\author{
Jing Sun \\ Department of Naval Architecture \\ and Marine Engineering \\ University of Michigan \\ Ann Arbor, Michigan 48109 \\ Email: jingsun@umich.edu
}

\author{
Huei Peng \\ Department of Mechanical Engineering \\ University of Michigan \\ Ann Arbor, Michigan 48109 \\ Email: hpeng@umich.edu
}

\begin{abstract}
Open-Circuit-Voltage $(\mathrm{OCV})$ is an essential part of battery models for state-of-charge (SOC) estimation. In this paper, we propose a new parametric OCV model, which considers the staging phenomenon during the lithium intercalation/deintercalation process. Results show that the new parametric model improves SOC estimation accuracy compared to other existing OCV models. Moreover, the model is shown to be suitable and effective for battery state-of-health monitoring. In particular, the new OCV model can be used for incremental capacity analysis (ICA), which reveals important information on the cell behavior associated with its electrochemical properties and aging status.
\end{abstract}

\section{INTRODUCTION}

With the widespread use of lithium-ion batteries for energy storage, the development of reliable and efficient battery management systems (BMS) has become a crucial task [1-3]. Two important functions of BMS are the state-of-charge (SOC) estimation and state-of-health $(\mathrm{SOH})$ determination $[4,5]$. SOC is commonly defined as "the percentage of the maximum possible charge that is present inside a rechargeable battery" (in this study, SOC is defined with respect to the current total capacity), and SOH is "a 'measure' that reflects the general condition of a battery and its ability to deliver the specified performance in comparison with a fresh battery" [6]. Typically, the quantitative definition of SOH is based either on the battery capacity or the internal resistance, depending on specific applications.

The on-line estimation of battery SOC has been studied ex-

\footnotetext{
*Address all correspondence to this author.
}

tensively in literature (see Ref. [7] and references therein). Most of those methods, including the extended Kalman filter (EKF) approach [8-10], require an accurate open-circuit-voltage (OCV) model which relates OCV to SOC $[7,11,12]$. The OCV-SOC function is implemented either as a look-up table or an analytical expression, while the latter has several advantages including computational efficiency (since no interpolation is needed) and ease for analysis [13].

Several analytical OCV models proposed in the literature are summarized in Ref. [13]. Those are phenomenological models built with curve fitting without considering the complex battery physical behavior during the lithium-ion intercalaction/deintercalation process $[14,15]$. However, as reported in our previous work [5], the OCV data obtained from the galvanostatic charging/discharging of batteries at low $\mathrm{C}$ rate displays voltage plateaus and transitions (please see the voltage curve plotted in Fig. 1) that correspond to the staging phenomenon at the graphite anode [5, 16-19]. Without proper parametrization, this critical phenomenon is usually lost when the OCV data is fitted with those existing models. At the same time, because of the wide flat region on the OCV-SOC curve, a small mismatch in OCV fitting may cause a large deviation when used for SOC estimation.

Moreover, OCV data often reflect battery aging and performance degradation [20]. Using the so-called incremental capacity analysis (ICA) technique [16, 21], which differentiates the battery charged capacity $(Q)$ with respect to the terminal voltage $(V)$ and transforms voltage plateaus on the $V-Q$ (voltage-charged versus capacity) curve into clearly identifiable $\mathrm{d} Q / \mathrm{d} V$ peaks on the incremental capacity (IC) curve (see Fig. 1), gradual changes in cell behavior can be detected, based on life cycle test data, 

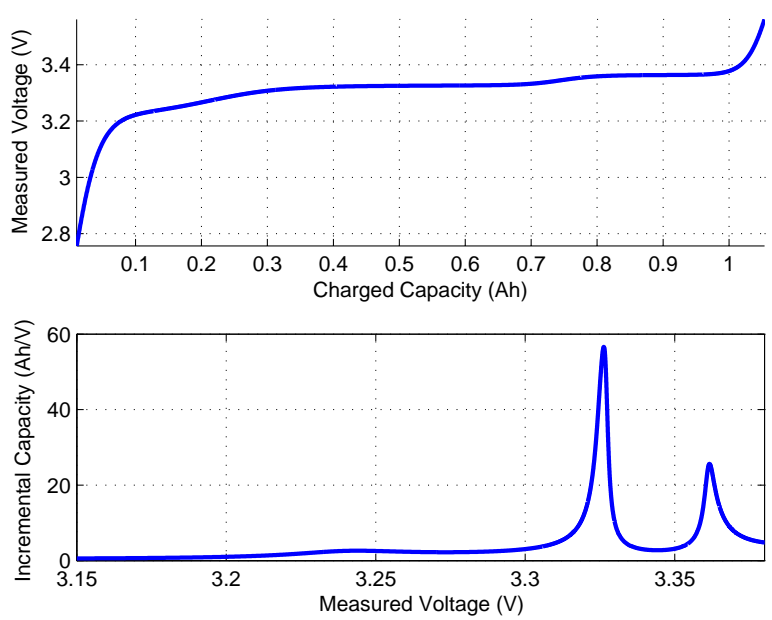

Figure 1. ICA PERFORMED ON CHARGING VOLTAGE CURVE AT $\frac{1}{20}$ C RATE

with greater sensitivity than those based on conventional methods [22]. One major challenge in ICA for battery aging analysis is its sensitivity to measurement noise. Since all the peaks on an IC curve lie within the flat region of the OCV curve, computing the derivatives directly from the data set often leads to inaccurate and undesirable results, even after careful data filtering. By designing an OCV model that captures those voltage plateaus and the subtle transitions over the flat region, one can perform ICA without using numerical derivative to study battery performance, thereby revealing useful information associated with its electrochemical properties and aging status [22,23]. Therefore, for the purposes of both SOC estimation and $\mathrm{SOH}$ monitoring through ICA, an OCV model that can capture the underlying physical process over the flat region and be capable of supporting ICA is needed.

In this paper, we propose a new parametric OCV model for lithium-ion batteries that considers the staging phenomenon during the lithium intercalation/deintercalation process. The model is intended for both SOC estimation and $\mathrm{SOH}$ monitoring. The study is based on a battery life cycle test data set collected from eight different $\mathrm{A} 123 \mathrm{LiFePO}_{4}$ cells over a period of 18 months.

The remainder of this paper is organized as follows. First, the development and parametrization of the new OCV model is proposed. Then we compare the new OCV model with other existing models in terms of OCV data fitting and SOC estimation accuracy. After that we elucidate the application of the new OCV model for $\mathrm{SOH}$ monitoring, followed by the conclusions.

\section{A NEW OPEN-CIRCUIT-VOLTAGE MODEL}

We propose a new OCV model structure that can capture the lithium-ion intercalaction/deintercalation process while fitting the OCV-SOC data. Instead of measuring the OCV after a long relaxation period at different SOC levels that span the en- tire operating range, the data used for the development of the our OCV model are collected by charging/discharging a $\mathrm{LiFePO}_{4}$ battery cell at $\frac{1}{20} \mathrm{C}$ rate. The upper and lower cut-off voltage limits are $3.6 \mathrm{~V}$ and $2.1 \mathrm{~V}$ respectively.

The total charge stored between the upper and lower limits is defined as the battery total capacity. The voltage data obtained by slow charging/discharging reflects the OCV at a close-toequilibrium status [21]. Although the close-to-equilibrium OCV curve shown in Fig. 2 may be affected by hysteresis and diffusion voltage due to lack of relaxation, it can sufficiently represent the generic electrochemical properties that can be used for ICA and quantifying capacity fading as presented in Ref. [21]. For future studies, more accurately measured OCV data may be used, and close-to-equilibrium OCV data collected at different current rates may be compared.

\section{Parametrization}

Based on the studies presented in Refs. [5, 16, 21], there are mainly three observable voltage plateaus and two transitions over the flat area on the OCV curve of $\mathrm{LiFePO}_{4}$ battery cells. In our model, those nonlinear characteristics are captured by the following sigmoid functions,

$$
\begin{aligned}
O C V(z)= & K_{0}+K_{1} \frac{1}{1+e^{\alpha_{1}\left(z-\beta_{1}\right)}}+K_{2} \frac{1}{1+e^{\alpha_{2}\left(z-\beta_{2}\right)}} \\
& +K_{3} \frac{1}{1+e^{\alpha_{3}(z-1)}}+K_{4} \frac{1}{1+e^{\alpha_{4} z}}+K_{5} z
\end{aligned}
$$

where $z$ is the SOC, $K_{0 \sim 5}$ are the linear parameters, and $\alpha_{1 \sim 4}$ and $\beta_{1 \sim 2}$ are the nonlinear parameters. This parametrization shares some common features with the electrochemical model of $\mathrm{LiFePO}_{4}$ proposed in Ref. [15]. More specifically, our model can be interpreted as a reduced-order composition and generalization of the cathode and anode OCV functions in Ref. [15] (where hyperbolic and exponential functions are used).

As shown in Fig. 2, the collected charging/discharging data are averaged and fitted with OCV model in Eq. (1). The parameters are determined using the Matlab curve fitting toolbox. The root mean square (rms) value of the fitting error is $2.3 \mathrm{mV}$. Since in practice most of the battery operations are in the middle SOC range, we may focus the fitting over SOC from $10 \%$ to $90 \%$ so that the model accuracy could be further improved by excluding the highly nonlinear area at both high and low SOC regimes. The corresponding results are plotted in Fig. 3, where the rms value of the fitting error is reduced to $1 \mathrm{mV}$ and the maximum fitting error is less than $2.5 m \mathrm{~V}$.

\section{Comparison with Existing Models}

The data fitting accuracy of our new OCV model is compared with the five different models summarized in Ref. [13], where the polynomial model is reported to be the most accurate. Those models are reorganized and listed in Tab. 1. For 


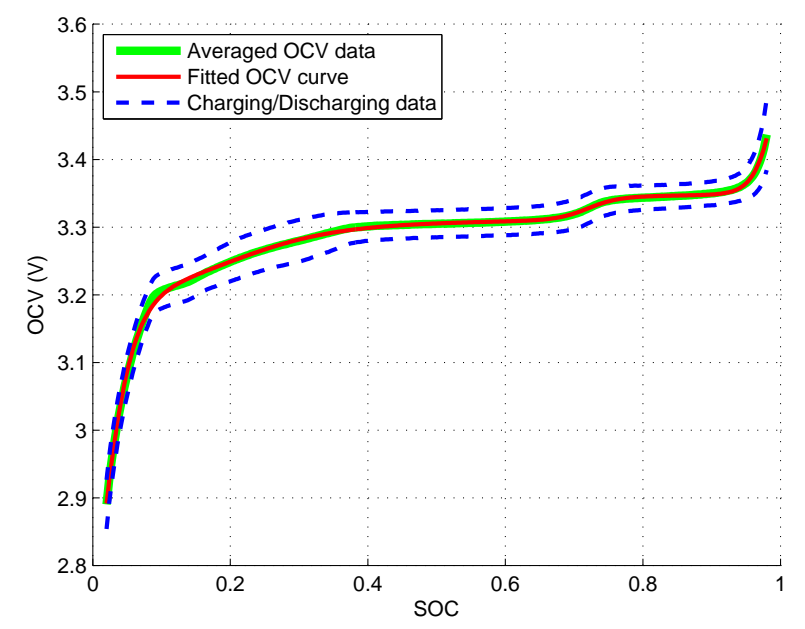

Figure 2. FITTING RESULT WITH THE PROPOSED OCV MODEL

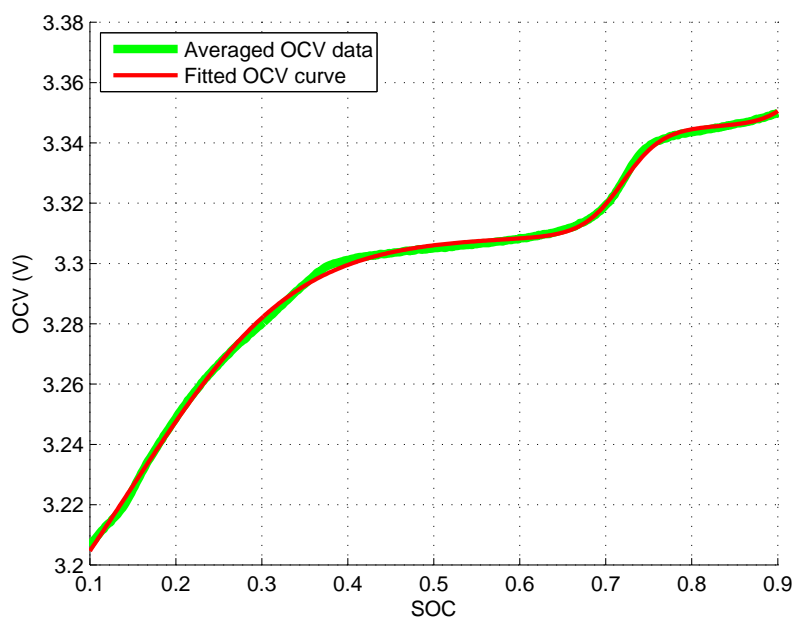

Figure 3. OCV MODEL FITTED OVER SOC FROM $10 \%$ to $90 \%$

our own comparison, the OCV data presented in Fig. 3 is then fitted with those models using Matlab curve fitting toolbox, and the results with their rms errors and maximum errors are shown and summarized in Fig. 4 and Tab. 2. One can see that the new OCV model proposed in Eq. 1 has better fitting accuracy than all those five models. Consequently, by incorporating the new OCV model, improvement in the SOC estimation results may also be expected.

It should be noted that none of the other five OCV models listed in Tab. 1 is suitable for IC analysis. A plot of IC curves based on different OCV fitting results is shown in Fig. 5. It can be observed that the new model (model \#6) can capture the two IC peaks associated with staging whereas all other models only show one peak in the IC curves. Therefore, model \#6 can extract more aging information from the OCV data compared to other
Table 1. OCV MODELS SUMMARIZED IN REF. [13] TOGETHER WITH THE NEW MODEL

\begin{tabular}{lllc}
\hline$\#$ & & OCV Models & Ref. \\
\hline 1 & $O C V(z)=$ & $K_{0}-\frac{K_{1}}{z}-K_{2} z+K_{3} \ln (z)+K_{4} \ln (1-z)$ & {$[9]$} \\
2 & $O C V(z)=$ & $K_{0}+K_{1}\left(1-e^{-\alpha_{1} z}\right)+K_{2}\left(1-e^{\left.-\frac{\alpha_{2}}{1-z}\right)+K_{3} z}\right.$ & {$[24]$} \\
3 & $O C V(z)=$ & $K_{0}+K_{1} e^{-\alpha_{1}(1-z)}-\frac{K_{2}}{z}$ & {$[25]$} \\
4 & $O C V(z)=$ & $K_{0}+K_{1} e^{-\alpha_{1} z}+K_{2} z+K_{3} z^{2}+K_{4} z^{3}$ & {$[26]$} \\
5 & $O C V(z)=$ & $K_{0}+K_{1} z+K_{2} z^{2}+K_{3} z^{3}+K_{4} z^{4}+K_{5} z^{5}+K_{6} z^{6}$ & {$[27]$} \\
6 & $O C V(z)=$ & $K_{0}+K_{1} \frac{1}{1+e^{\alpha_{1}\left(z-\beta_{1}\right)}}+K_{2} \frac{1}{1+e^{\alpha_{2}\left(z-\beta_{2}\right)}}$ & Proposed \\
& & $+K_{3} \frac{1}{1+e^{\alpha_{3}(z-1)}}+K_{4} \frac{1}{1+e^{\alpha_{4} z}}+K_{5} z$ & model \\
\hline
\end{tabular}

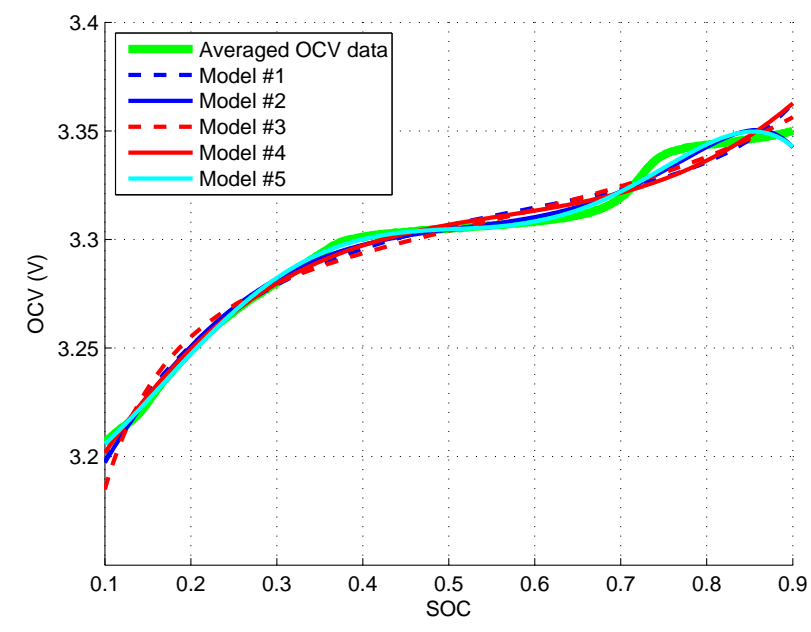

Figure 4. COMPARISON OF OCV FITTING RESULTS

models and will be used for further analysis in $\mathrm{SOH}$ monitoring. For models \#2 and \#5, $\mathrm{d} Q / \mathrm{d} V$ is not defined at 3.35 volt because of a singularity in the math expression. Consequently, spikes in both directions are shown in Figure 5 for those two models' IC curves.

\section{STATE-OF-CHARGE ESTIMATION BASED ON EX- TENDED KALMAN FILTER}

The extended Kalman filter (EKF) based approach discussed in Ref. [10] is used to illustrate the implementation of the new OCV model in SOC estimation. The battery test data for this study is collected through the experimental set-up introduced in Ref. [28] and the first-order RC model is used for the estimation 
Table 2. FITTING RESULTS OF OCV MODELS FROM TAB. 1

\begin{tabular}{ccc}
\hline Model \# & RMS Error $(\mathrm{mV})$ & Max Error $(\mathrm{mV})$ \\
\hline 1 & 5.2 & 12.6 \\
2 & 3.1 & 8.9 \\
3 & 5.6 & 21.3 \\
4 & 4.7 & 12.7 \\
5 & 2.1 & 7.3 \\
6 & 1.0 & 2.5 \\
\hline
\end{tabular}

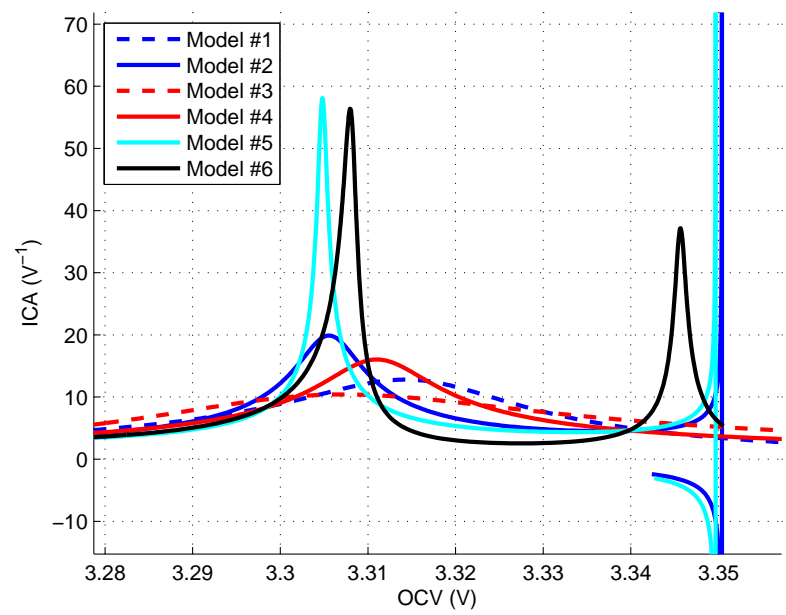

Figure 5. COMPARISON OF IC CURVES FROM FITTED OCV

as follows,

$$
\begin{aligned}
z_{k+1} & =z_{k}-\left(\frac{\eta_{i} \Delta t}{Q_{c}}\right) I_{k}, \\
V_{r c, k+1} & =\exp \left(\frac{-\Delta t}{R_{1} C}\right) V_{r c, k}+R_{1}\left[1-\exp \left(\frac{-\Delta t}{R_{1} C}\right)\right] I_{k} \\
V_{k} & =O C V\left(z_{k}\right)+V_{r c, k}+R_{0} I_{k}
\end{aligned}
$$

where $z_{k}$ is the SOC, $\eta_{i}$ is the charging/discharing efficiency, $\Delta t$ is the time step, $Q_{c}$ is the battery capacity, $I_{k}$ is the input current, $V_{k}$ is the total terminal voltage, $V_{r c, k}$ is voltage of the RC circuit, $R_{0}, R_{1}$ and $C$ are battery internal resistance and capacitance [28]. The OCV function is represented by the model proposed in Eq. (1). The first-order RC model is used here for simplicity, as the model accuracy in SOC estimation has been shown in [28]. One can certainly improve the results further by using other models with higher orders at the expense of additional computation and complexity.

In this study, all the parameters in the RC model are set to
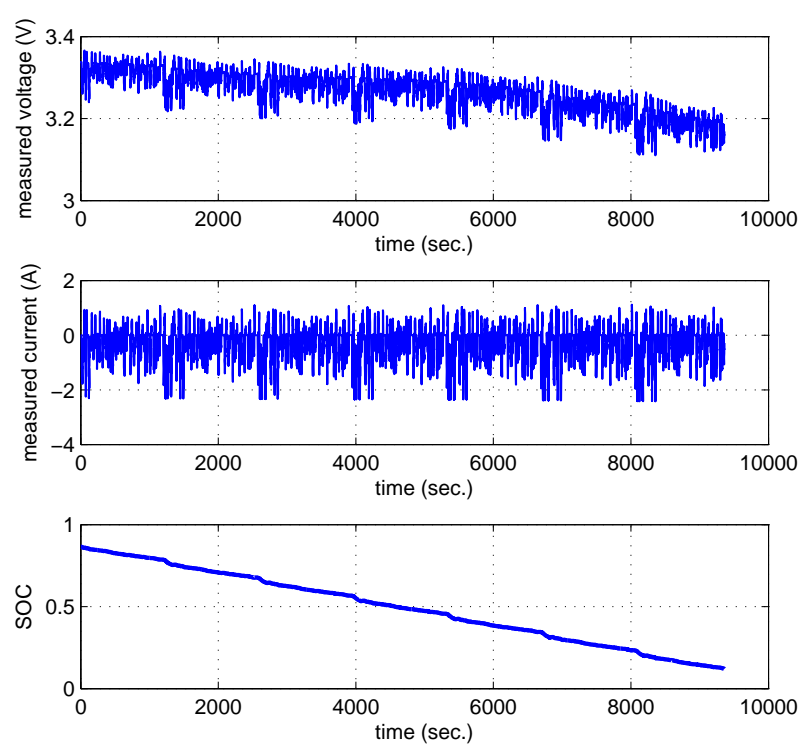

Figure 6. BATTERY DATA TESTED UNDER FUDS

be constants without performing iterative optimizations as discussed in Ref. [13].

The battery data tested under the Federal Urban Driving Schedule (FUDS) is shown in 6. Same as in Ref. [10], the SOC data obtained by Coulomb counting in the Arbin battery test bench, where high precision current sensor is used, is assumed to be the "true" SOC and used as a reference for performance evaluation. Therefore the SOC estimation error is defined as follows,

$$
e_{S O C}=\mathrm{SOC}_{e s t}-\mathrm{SOC}_{d a t a}
$$

where $e_{S O C}$ is the SOC estimation error, $\mathrm{SOC}_{\text {est }}$ is the $\mathrm{SOC}$ estimated by the EKF algorithm and $\mathrm{SOC}_{\text {data }}$ is the reference $\mathrm{SOC}$ calculated from the measured data shown in Fig. 6. Please note that the SOC from Arbin Coulomb counting, while representing the best reference that we can get, is only approximately accurate due to the unavoidable error accumulation in Coulomb counting [10].

The model parameters used in the EKF based SOC estimation are shown in Tab. 3.

A detailed description of the EKF algorithm can be found in both Refs. [10] and [13]. The parameters of the EKF are calibrated by trial and error using the data collected in our aging tests, and exactly same parameters (listed in Tab. 4) are used in the following study for models \#5 and \#6.

Figure 7 displays the EKF based SOC estimation results with the new model \#6 and the polynomial model \#5. The two plots represent two cases with different initial SOC error $(+10 \%$ and $-10 \%$ respectively). One can see that the estimated SOC with both models converges into the 5\% estimation error band, which 
Table 3. PARAMETERS OF MODELS \#5 and \#6 IN SOC ESTIMATION

\begin{tabular}{ccc}
\hline Model Parameters & Model \#5 & Model \#6 \\
\hline $\mathrm{K}_{0}$ & 3.0896 & 3.4002 \\
$\mathrm{~K}_{1}$ & 1.1627 & 0.0080 \\
$\mathrm{~K}_{2}$ & -2.3821 & 0.0785 \\
$\mathrm{~K}_{3}$ & 2.1870 & -0.2150 \\
$\mathrm{~K}_{4}$ & -0.5444 & -1.3032 \\
$\mathrm{~K}_{5}$ & -0.1939 & 0.0891 \\
$\mathrm{~K}_{6}$ & 0.0582 & N/A \\
$\alpha_{1}$ & N/A & -14 \\
$\alpha_{2}$ & N/A & -18 \\
$\alpha_{3}$ & N/A & 28 \\
$\alpha_{4}$ & N/A & 40 \\
\hline
\end{tabular}

Table 4. PARAMETERS OF EKF BASED SOC ESTIMATOR

\begin{tabular}{cc}
\hline Parameters & Values \\
\hline Error covariance of process noises, Q & {$\left[\begin{array}{cc}0.15 & 0 \\
0 & 1\end{array}\right]$} \\
Error covariance of observation noises, $\mathrm{V}$ & 0.01 \\
\hline
\end{tabular}

are considered to be acceptable SOC estimation accuracy [13]. In particular, with positive initial error, the SOC estimated with the new model converges much faster than the SOC estimated with the polynomial model. This difference in convergence rate with positive initial error can be explained by the curve fitting results shown in Figs. 3 and 4, where model \#6 have better accuracy than \#5 in the high SOC region. On the other hand, both models have approximately the same accuracy when SOC is below 70\% and therefore have similar convergence rate with negative initial error. Please note that the $5 \%$ error bound can be achieved with other initial error (e.g., $20 \%$ or $30 \%$ ).

In summary, the new OCV model performs well when used in EKF based SOC estimation. Without extensive calibration and training, the model still delivers an accurate estimation result with less than $5 \%$ errors. The results presented in this section demonstrates the potential of implementing this new OCV model for SOC estimation problems.
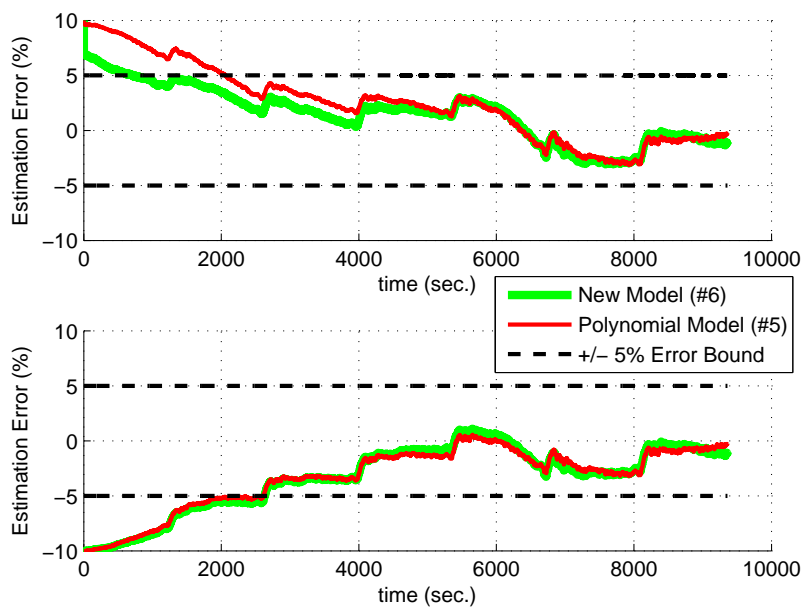

Figure 7. COMPARISON OF SOC ESTIMATION ERROR
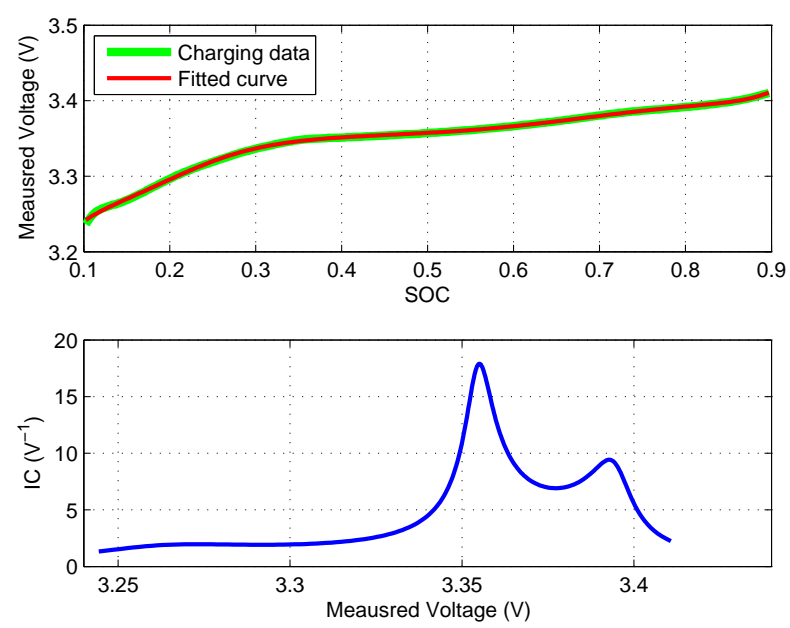

Figure 8. V-SOC AND IC CURVE FROM $\frac{1}{2}$ C CHARGING DATA

\section{STATE-OF-HEALTH MONITORING BASED ON INCRE- MENTAL CAPACITY ANALYSIS}

In addition to its advantage in SOC estimation, the potential of the new model in $\mathrm{SOH}$ monitoring will be illustrated within the ICA framework. Moreover, it will be shown that the proposed parametrization can be used to capture the intercalation process with both OCV data and normal charging data. Even though ICA was originally proposed for "close-to-equilibrium" conditions, it was shown in Ref. [5] that the peaks on the IC curve can also be identified with normal charging data. Hence, the results presented in this section for ICA are all based on $\frac{1}{2} \mathrm{C}$ battery charging data (same as in Ref. [5]).

As shown in Fig. 8, where the charging data is fitted with the model given by Eq. (1) with OCV replaced by the terminal voltage, we can obtain the IC curve directly by taking the reciprocal 


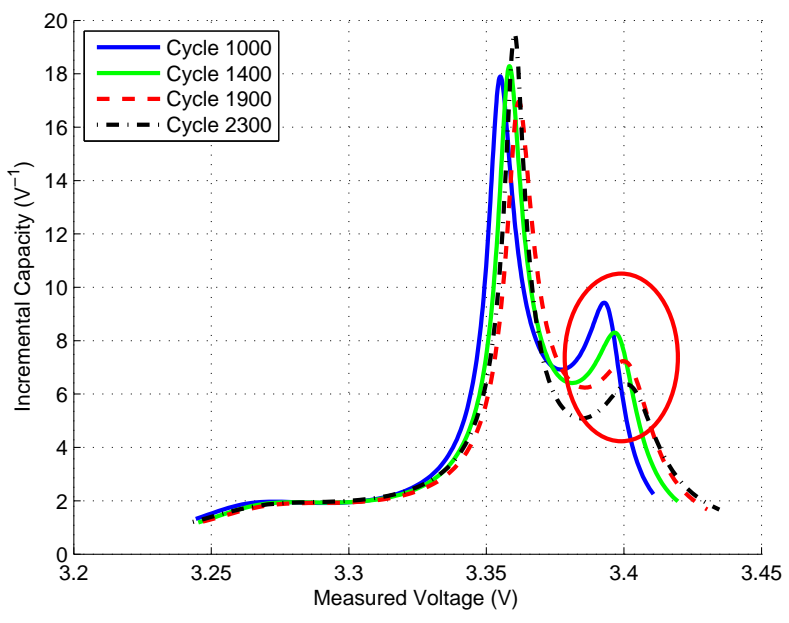

Figure 9. IC CURVES OF DATA AT DIFFERENT AGES
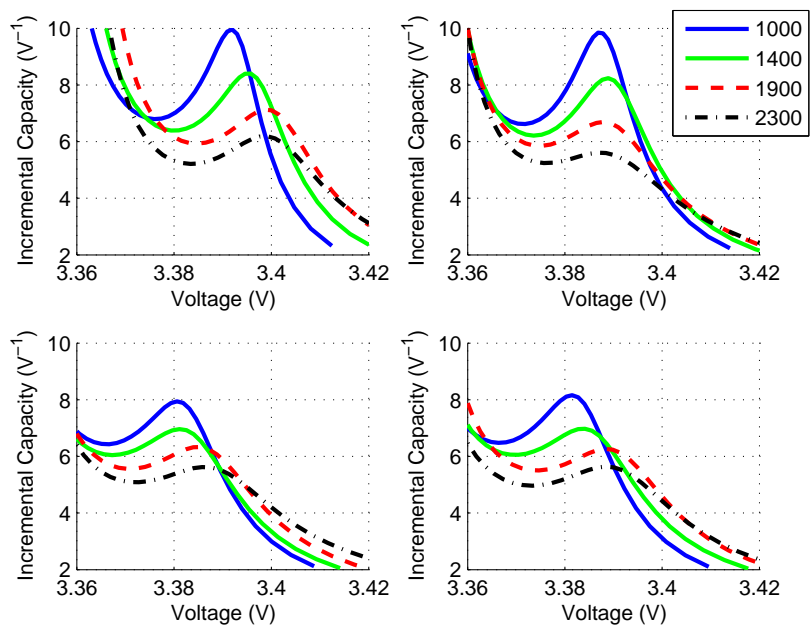

Figure 10. IC CURVES COMPARISON FROM FOUR DIFFERENT CELLS

after differentiating the analytical V-SOC function.

\section{Incremental Capacity Analysis at Different Aging Stages}

The IC curves of battery charging data collected at different aging cycles under $35^{\circ} \mathrm{C}$ environment are shown in Fig. 9. The numerical values in the plot legends represent the aging cycle number. The IC peaks in the circled area in Fig. 9 clearly shows a monotonic decreasing trend, which implies a battery degradation that is mainly related to the loss of active material at the graphite anode $[16,21]$.

The IC curves from four different $\mathrm{A} 123 \mathrm{LiFePO}_{4}$ cells are plotted in Fig. 10, where only the second peaks correlated to aging are shown for comparison. The curves show that the aging

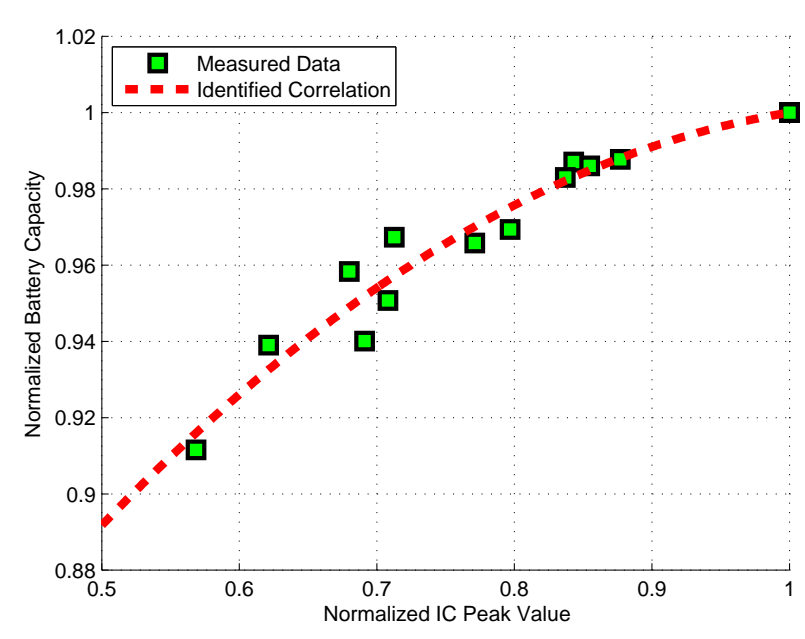

Figure 11. CORRELATION BETWEEN BATTERY CAPACITY AND IC PEAK VALUES

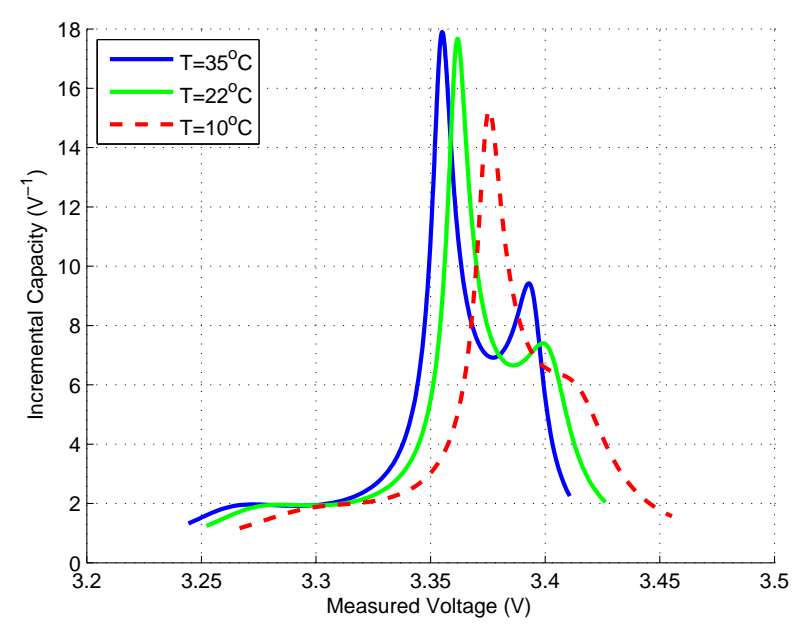

Figure 12. IC CURVES OF DATA AT DIFFERENT TEMPERATURE

phenomenon identified from IC peak changes is common for the batteries tested in our experiment. Those peak values are then quantified and correlated with the faded cell capacity in Fig. 11. The close correlation between the cell capacity fading and IC peak revealed by the new parametric model in the form of Eq. (1) can therefore be used for battery SOH monitoring. Moreover, even though the quantitative relation identified in this work have not been demonstrated to work on other types of cells, we believe that the procedure and approaches reported in this paper are general and should be applicable to many other Li-ion battery systems. 

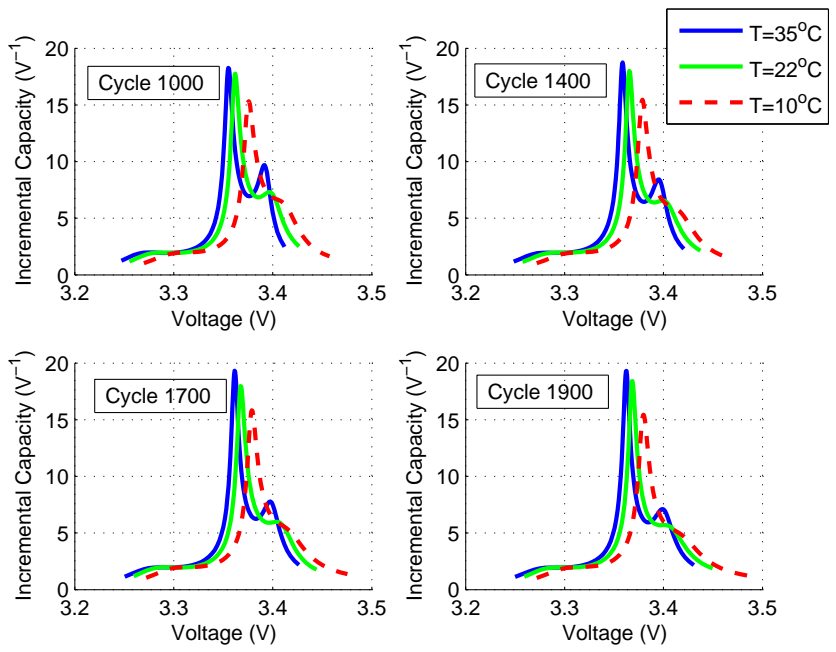

Figure 13. IC CURVES AT DIFFERENT TEMPERATURE AND AGES

\section{Incremental Capacity Analysis at Different Tempera- ture}

Test data were also collected at different temperature throughout battery aging process. ICA was performed with respect to different temperature to evaluate and confirm the sensitivity to temperature and results are presented in Fig. 12. The intensities of the IC peaks reduce as temperature drops, which may be attributed to the slow lithium-ion diffusion or lithium plating phenomenon at low temperature as elucidated in Ref. [29]. When the temperature is too low, say at $10^{\circ} \mathrm{C}$, the second peak could disappear from the IC curve.

Figure 13 presents the IC curves at different temperature and different aging stages. A temperature dependence of the IC peaks and the model parameters may be evaluated. The first peak on these four plots shows consistent temperature sensitivity while the second peak shows the aging status. The test data corresponding to higher temperature will be more reliable for ICA and capacity fading identification.

\section{Summary of the Comparisons}

The two comparisons of IC curves obtained from the new OCV parametric model illustrates the utility of the model in the analysis of battery $\mathrm{SOH}$ from the electrochemical perspective. Compared to those data analysis approaches discussed in Ref. [5], this parametric model based method is more straightforward and easier for implementation. ICA can be performed without extra data processing other than the curve fitting. Therefore the potential application of this model is not limited to SOC estimation, it is also a convenient tool for studying battery aging and performance degradation.

\section{CONCLUSIONS}

In this paper, we propose a new OCV parametric model, which considers the staging phenomenon during the lithium intercalation/deintercalation process in selecting the parametric form, for lithium-ion batteries. The special parametrization not only leads to better fitting accuracy when applied to experimental battery OCV data compared to existing models, but also yields natural IC characteristics that other models fail to represent. The new parametric model is applied, together with an EKF, to SOC estimation and its effectiveness is demonstrated on FUDS cycle data. Moreover, we show that the parametric model can also be applied for ICA based SOH monitoring. Through comparisons of IC curves, the model is shown to be capable of capturing battery cell's electrochemical properties at different operating temperature conditions and aging stages. Future research will investigate the sensitivity and robustness of the proposed model structure. A hybrid model that combines the simpler models (i.e., \#1 5) and the proposed model \#6 may be explored for further improvement of the ICA results. Efforts will also be directed towards characterizing the correlations between the model parameters and the degradation of battery performance, and developing more efficient algorithms for the determination of the OCV model parameters.

\section{ACKNOWLEDGMENT}

This material is based upon work supported by the Department of Energy under Award Number DE-PI0000012.

\section{REFERENCES}

[1] Armand, M., and Tarascon, J. M., 2008. "Building better batteries". Nature, 451, pp. 652-657.

[2] Dunn, B., Kamath, H., and Tarascon, J.-M., 2011. "Electrical energy storage for the grid: A battery of choices". Science, 6058, pp. 928-935.

[3] Roscher, M. A., and Sauer, D. U., 2011. "Dynamic electric behavior and open-circuit-voltage modeling of $\mathrm{LiFePO}_{4}$ based lithium ion secondary batteries". J. Power Sources, 196, pp. 331-336.

[4] Meissner, E., and Richter, G., 2003. "Battery monitoring and electrical energy management precondition for future vehicle electric power systems". J. Power Sources, 116, pp. 79-98.

[5] Weng, C., Cui, Y., Sun, J., and Peng, H., 2013. "On-board state of health monitoring of lithium-ion batteries using incremental capacity analysis with support vector regression". J. Power Sources, 235, pp. 36-44.

[6] Pop, V., Bergveld, H. J., Danilov, D., Regtien, P. P. L., and Notten, P. H. L., 2008. Battery Management Systems: Accurate State-of-Charge Indication for Battery-Powered Applications, 1st ed. Springer.

[7] Piller, S., Perrin, M., and Jossen, A., 2001. "Methods for 
state-of-charge determination and their applications". $J$. Power Sources, 96, pp. 113-120.

[8] Plett, G. L., 2004. "Extended kalman filtering for battery management systems of LiPB-based hev battery packs, part 1. background". J. Power Sources, 134, pp. 252-261.

[9] Plett, G. L., 2004. "Extended kalman filtering for battery management systems of LiPB-based hev battery packs, part 2. modeling and identification". J. Power Sources, 134, pp. 262-276.

[10] Plett, G. L., 2004. "Extended kalman filtering for battery management systems of LiPB-based hev battery packs, part 3. state and parameter estimation". J. Power Sources, 134, pp. 277-292.

[11] Santhanagopalan, S., and White, R. E., 2006. "Online estimation of the state of charge of a lithium ion cell". J. Power Sources, 161, pp. 1346-1355.

[12] Verbrugge, M., and Tate, E., 2004. "Adaptive state of charge algorithm for nickel metal hydride batteries including hysteresis phenomena". J. Power Sources, 126, pp. 236-249.

[13] Hu, X., Li, S., Peng, H., and Sun, F., 2012. "Robustness analysis of state-of-charge estimation methods for two types of li-ion batteries". J. Power Sources, 217, pp. 209219.

[14] Safari, M., and Delacourt, C., 2011. "Mathematical modeling of lithium iron phosphate electrode: Galvanostatic charge/discharge and path dependence". J. Electrochem. Soc., 158(2), pp. A63-A73.

[15] Safari, M., and Delacourt, C., 2011. "Modeling of a commercial graphite/LiFePO 4 cell”. J. Electrochem. Soc., 158(5), pp. A562-A571.

[16] Groot, J., 2012. "State-of-health estimation of li-ion batteries: Cycle life test methods". Master's thesis, Chalmers University of Technology.

[17] Yazami, R., and Touzain, P., 1983. "A reversible graphitelithium negative electrode for electrochemical generators". J. Power Sources, 9, pp. 365-371.

[18] Dahn, J. R., 1991. "Phase diagram of $\mathrm{Li}_{x} \mathrm{C}_{6}$ ". Phys. Rev., B44, pp. 9170-9177.

[19] Yazami, R., and Reynier, Y., 2006. "Thermodynamics and crystal structure anomalies in lithium-intercalated graphite". J. Power Sources, 153, pp. 312-318.

[20] Roscher, M. A., Assfalg, J., and Bohlen, O. S., 2011. "Detection of utilizable capacity deterioration in battery systems". IEEE Trans. Veh. Technol., 60(1), pp. 98-103.

[21] Dubarry, M., Svoboda, V., Hwu, R., , and Liaw, B. Y., 2006. "Incremental capacity analysis and close-to-equilibrium ocv measurements to quantify capacity fade in commercial rechargeable lithium batteries". Electrochem. Solid St., 9(10), pp. A454-A457.

[22] Liaw, B. Y., and Dubarry, M., 2010. "A roadmap to understand battery performance in electric and hybrid vehicle operation". In Electric and Hybrid Vehicles: Power Sources, Models, Sustainability, Infrastructure and the
Market, G. Pistoia, ed. Elsevier, pp. 375-403.

[23] Dubarry, M., and Liaw, B. Y., 2009. "Identify capacity fading mechanism in a commercial $\mathrm{LiFePO}_{4}$ cell". J. Power Sources, 194, pp. 541-549.

[24] Hu, Y., Yurkovich, S., Guezennec, Y., and Yurkovich, B., 2011. "Electro-thermal battery model identification for automotive applications". J. Power Sources, 196, pp. 449457.

[25] Neumann, D. E., and Lichte, S., 2011. "A multidimensional battery discharge model with thermal feedback applied to a lithium- ion battery pack". In NDIA Ground Vehicle Systems Engineering and Technology Symposium.

[26] Chen, M., and Rincón-Mora, G. A., 2008. "Accurate electrical battery model capable of predicting runtime and I-V performance". IEEE Trans. Veh. Technol., 21(2), pp. 504511.

[27] Szumanowski, A., and Chang, Y., 2008. "Battery management system based on battery nonlinear dynamics modeling”. IEEE Trans. Veh. Technol., 57(3), pp. 1425-1432.

[28] Hu, X., Li, S., and Peng, H., 2012. "A comparative study of equivalent circuit models for li-ion batteries". J. Power Sources, 198, pp. 359-367.

[29] Vetter, J., Novák, P., Wagner, M., Veit, C., Möller, K.-C., Besenhard, J., Winter, M., Wohlfahrt-Mehrens, M., Vogler, C., and Hammouched, A., 2005. "Ageing mechanisms in lithium-ion batteries”. J. Power Sources, 147, pp. 269-281. 